\title{
Essentials of Trauma Anesthesia, Second Edition
}

\author{
Albert J. Varon, Charles E. Smith (Editors). Cambridge University Press, 2018. ISBN \\ 978-1-3166-3671-8
}

\author{
Chadi Saliba, MD $\cdot$ Doreen Yee, MD
}

Received: 19 July 2018/Accepted: 19 July 2018/Published online: 10 August 2018

(C) Canadian Anesthesiologists' Society 2018

As trauma anesthesia is now an essential subspecialty in the field of anesthesiology, Essentials of Trauma Anesthesia, Second Edition should prove invaluable to all trainees and serve as a reference in an acute care setting. Co-editors Varon and Smith have organized the second edition of this textbook into three major sections: Core Principles in Trauma Anesthesia, Anesthetic Considerations for Trauma, and Anesthetic Management in Special Trauma Populations. Chapters in the three sections are uniformly organized with an introduction, multiple subheadings, a key points summary, and references for additional reading. It is easy to read, is well organized, and focuses on the practical aspects of trauma anesthesia.

The first section includes 12 comprehensive chapters. The discussion in the first chapter regarding trauma epidemiology is particularly helpful as it gives a comprehensive overview of the overall incidence of trauma along with multiple statistical studies and mortality data. Care for the trauma patient starts at the prehospital level where a patient's initial evaluation, triage, and resuscitation takes place. Because optimal care of a severely injured patient requires teamwork, the "ABCDEs of trauma" will help familiarize care providers (who may not have taken the Advanced Trauma and Life Support course) with the principles of primary and secondary surveys, initial evaluation, and management. These components are discussed in detail in Chapters 3 through 6 .

C. Saliba, MD · D. Yee, MD ( $₫)$

Department of Anesthesia, University of Toronto, Toronto, ON, Canada

e-mail: Doreen.Yee@sunnybrook.ca
Managing the airway is critical in the trauma setting. A summary of the indications for acquiring a definitive airway, the needed equipment and medications (with their contraindications), as well as maintaining cervical spine immobilization, enables each member of the trauma team to follow these primary steps in the evaluation and management of the airway. The modified American Society of Anesthesiologists' difficult airway algorithm for trauma and indications for a surgical airway are useful for organizing a backup plan.

As patients' resuscitation is an important aspect of anesthesia practice, the section addressing this specific issue is particularly important. Chapter 4 is dedicated to shock, resuscitation and fluid therapy, and Chapters 5 and 6 focus on vascular cannulation, blood component therapy, and trauma coagulopathy. Shock classification is outlined and includes the pathophysiology, recognition, and diagnosis. The possible responses to initial fluid resuscitation are described, with an emphasis on fast and slow bleeders. Subjects such as fluid alternatives for smallvolume resuscitation, the early resuscitation phase, damage control, hemostatic resuscitation, and the late resuscitation phase are discussed and supported by clear clinical evidence. Understanding and applying these concepts to resuscitation of the trauma patient constitute a key element in best practice techniques.

The chapter on general anesthesia for trauma focuses on several high-yield topics, including the preoperative period, operating room setup, interaction between the pharmacology of anesthetic drugs, and the pathophysiology of the polytrauma patient. There is also a focus on hypothermia, its complications, and the various methods of patient rewarming. These topics are then summarized in tables, which are highly practical and easy to remember. 
Regional anesthesia and its application in trauma patients are well described, with a focus on contraindications, coagulation profiles, and various ultrasonographic approaches.

Monitoring trauma patients is crucial, as their conditions may change rapidly. An excellent description of various systems, their applications, indications, and contraindications are featured in Chapter 9 of this second edition of Essentials of Trauma Anesthesia, which focuses on the lactate level and the base deficit as markers of tissue perfusion and oxygen debt. The use of bedside echocardiography and, ultimately, point-of-care ultrasonography, as an evolving diagnostic and monitoring modality, is discussed in Chapter 10. The coagulopathy of trauma poses a unique challenge, and we found Chapter 11 (Coagulation Monitoring of the Bleeding Trauma Patient) essential and comprehensive in this regard. The concepts of the coagulation system and the assessment and treatment of coagulopathy in trauma patients are well described. The postoperative care of the trauma patient is fully addressed and includes multiple key points.

In the second section of this book, anesthetic considerations are outlined for cases of specific injuries in certain systems or areas. For example, perioperative priorities are discussed for adult patients with traumatic brain and spinal cord injury. Specific concerns are also presented for patients with ocular, maxillofacial, chest, abdominal, and musculoskeletal injuries. This section is an excellent reference for anesthesia providers caring for multi-system trauma patients.

Lastly, the final section of Essentials provides a good description of the perioperative considerations in special trauma populations - e.g., altered pharmacokinetics and special fluid replacement algorithms in burn patients along with a detailed approach for airway management. The Pediatric Trauma chapter offers a comprehensive table of appropriately sized resuscitative equipment for these young patients as well as application of the modified Glasgow Coma Scale. It also includes the challenges encountered when imaging the child's cervical spine.

As the incidence of geriatric trauma is on the rise, this special group with its age-related changes and decreased physiologic reserve, as well as its associated use of polypharmacy, anticoagulation, and subtler presentations of occult shock, are highlighted in a single chapter. Finally, pregnant trauma patients, albeit less common, pose unique considerations with regard to the management of two patients (i.e., mother and fetus): specific Advanced Trauma and Life Support considerations and working as a large multi-disciplinary team.

In summary, Essentials of Trauma Anesthesia, Second Edition is effectively organized and well referenced. Trainees interested in trauma anesthesia would benefit from reading it cover to cover as it could help them acquire a firm understanding of best practices in trauma anesthesia. The Key Points summaries are helpful for an overview of the subspecialty area. This textbook is also recommended for any anesthesiologist who has the lead role in trauma resuscitations.

Conflicts of interest None declared.

Editorial responsibility This submission was handled by Dr. Hilary P. Grocott, Editor-in-Chief, Canadian Journal of Anesthesia. 